\title{
Annealing effect on the structure characteristics of nano-scale damascene copper lines
}

\author{
T. Konkova ${ }^{1,2}$, S. Mironov ${ }^{3}$, Y. Ke ${ }^{4}$, J. Onuki $^{1}$ \\ ${ }^{1}$ Department of Materials Science and Engineering, Ibaraki University, Hitachi, Ibaraki 316-8511, Japan \\ ${ }^{2}$ Institute for Metals Superplasticity Problems of Russian Academy of Sciences, 39 Khalturin Str., Ufa, 450001, Russia \\ ${ }^{3}$ Department of Materials Processing, Graduate School of Engineering, Tohoku University, 6-6-02 Aramaki-aza-Aoba, Sendai \\ 980-8579, Japan \\ ${ }^{4}$ Graduate School of Science and Engineering, Ibaraki University, Hitachi, Ibaraki 316-8511, Japan \\ tnkonkova@mx.ibaraki.ac.jp; konkova_05@mail.ru
}

\begin{abstract}
High-resolution electron backscatter diffraction (EBSD) technique was applied for systematic and detailed study of grain structure and texture changes in various microstructural regions of nano-scale damascene copper lines after annealing in a wide temperature range of $200-500^{\circ} \mathrm{C}$. To ensure reliability of the obtained results, large EBSD maps including several thousand grains were obtained in each case. Above $200^{\circ} \mathrm{C}$, the grain structure was established to be surprisingly stable in both the overburden layer as well as within the lines. The grain growth in the lines was supposed to be suppressed by pinning effect of second-phase particles entrapped during electrodeposition process.
\end{abstract}

\section{Introduction}

Nowadays, the ongoing miniaturization of electronic devices is prompting a reduction of interconnect widths down to a nano-scale. For manufacturing of such interconnects, a copper damascene process is currently used. The significant problem is that resistivity of the narrow copper lines considerably increases in the nano-scale range [1-2]. This effect is usually attributed to electron scattering on sidewalls as well as on grain boundaries because grain size in such interconnects is expected to approach the mean electron free path ( $40 \mathrm{~nm}$ at ambient temperature). An evident way to minimize this problem is an appropriate annealing process which enables to produce a reasonably coarse-grained structure. In this regard, an annealing behavior of the electrodeposited copper becomes of particular practical interest.

The annealing behavior of the damascene copper lines appears to be a complex process. The grain growth is frequently found to initiate in the overburden layer [3-7], which presumably behaves as a thin film. Within the lines, however, the microstructure is surprisingly stable and grain coarsening here occurs only via penetration of the growing grains from the overburden [4-8]. It is also reported that the downward grain penetration may also be inhibited in the case of the very narrow lines [4-6].

High thermal stability of the microstructure within the lines is sometimes attributed to the pinning effect of secondphase particles entrapped during the electrodeposited process [4-6]. According to an alternative theory, the stabilization effect is related with a mismatch between thermal expansion of copper and silicon substrate which may produce significant thermal stresses during annealing [9-11]. However, both hypotheses are not experimentally verified yet.
Microstructural observations in the nano-scale wires are still limited. The above mentioned works have provided an important insight into the structural behavior of the damascene copper lines. It should be emphasized, however, that they were typically performed in a limited range of annealing conditions (including only one or two annealing temperatures) and were based on scanty experimental statistics. It is difficult to know, therefore, whether these results are isolated cases or representative examples. More systematic research is needed in this field with the final aim being precise microstructural control of the copper interconnects.

Attempting to establish a better understanding of the annealing processes and grain growth mechanism in these materials, this work presents a detailed study of grain structure and texture evolution in a wide temperature range.

\section{Experimental}

Damascene trenches (80 $\mathrm{nm}$ wide and $200 \mathrm{~nm}$ height) were patterned in $\mathrm{SiO}_{2} / \mathrm{Si}$ dielectric films using electron beam lithography and reactive ion etching. The reference directions employed in this study included longitudinal direction (LD) of the trenches, their transversal direction (TD) and normal direction (ND) of the silicon substrate. An ultra-thin TaN/Ta $(7.5 \mathrm{~nm} / 7.5 \mathrm{~nm})$ layer was first sputter-deposited on the trenches as a diffusion barrier and adhesion layer, followed by sputter deposition of a $50 \mathrm{~nm}$ copper seed layer to serve as the cathode for electroplating. To investigate the annealing behavior of the copper lines, the samples were annealed for 1 hour at a range of temperatures from 100 to $500^{\circ} \mathrm{C}$ in vacuum $\left(5 \times 10^{-5}\right.$ Torr) immediately after completion of the electrodeposition process. All samples were stored in vacuum prior to microstructural examinations. All microstructural observations were made on the longitudinal (i.e. LD-TD) plane at the trench heights of $\sim 50$ and $200 \mathrm{~nm}$ as well as in the overburden layer. The microstructural examinations were performed by using EBSD technique preceded by chemical mechanical polishing (CMP); a Hitachi S-4300SE field emission gun scanning electron microscope equipped with a TSL OIM ${ }^{\mathrm{TM}}$ EBSD system was employed. The EBSD maps were acquired with a scan step size of $30 \mathrm{~nm}$; a lower limit boundary misorientation cut-off of $2^{\circ}$ was used. Low-angle boundaries (LABs) and high-angle boundaries (HABs) were defined as those having misorientations below and above $15^{\circ}$, respectively. The $\Sigma 3$ twin boundaries were categorized as those having a $60^{\circ}<111>$ misorientation (within a Brandon's tolerance of $\left.\sim 8.7^{\circ}[12]\right)$. 
Due to lattice distortion and the fine nature of the microstructure, reliable EBSD maps could not be obtained from the as-deposited material as well as from the sample annealed at $100^{\circ} \mathrm{C}$. In these cases, the EBSD data were used only for qualitative evaluation of the developed microstructure.

\section{Results}

Grain structure

The EBSD maps illustrating effect of annealing temperature on grain structure in different parts of the damascene lines are shown in Fig. 1. In the maps, grains are colored according to their crystallographic orientations relative to the ND (color code triangle is given in the upper right corner of the figure); LABs and HABs are depicted as white and black lines, respectively. The grain size was quantified from the EBSD maps by measurement of grain area (ignoring twin boundaries) and calculation of the equivalent grain diameter assuming each grain as a circle (i.e., the so-called grain reconstruction method [13] was applied).

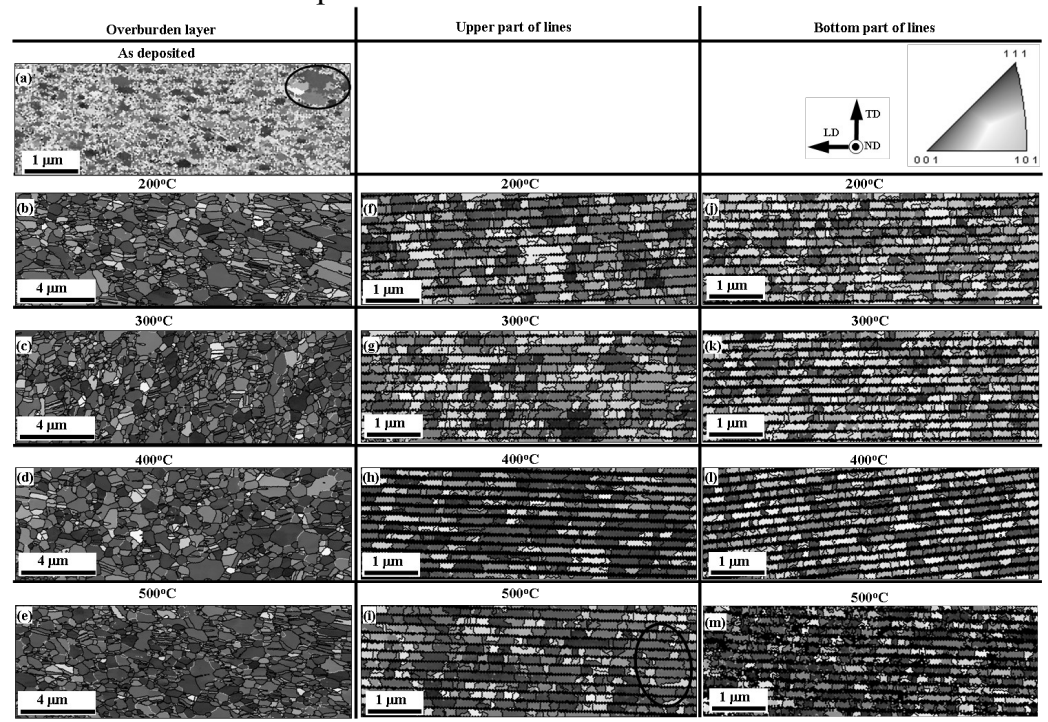

Fig. 1 EBSD maps illustrating effect of annealing temperature on grain structure in overburden layer (a-e), upper (f-i) and bottom (j-m) parts of damascene copper lines.

In the overburden layer, the as-deposited microstructure was comprised by fine elongated grains with a longitudinal size of $\sim 0.2-0.3 \mu \mathrm{m}$ and grain shape aspect ratio of $\sim 2$ (Fig. 1a). There were also isolated, relatively coarse $(\sim 1 \mu \mathrm{m})$ grains containing annealing twins in the microstructure (an example is encircled in Fig. 1a). These grains seemed to be recrystallized in appearance and presumably originated from the self-annealing effect. An annealing at $200^{\circ} \mathrm{C}$ led to significant microstructural changes in the overburden layer. The grain structure substantially coarsen (Fig. 1b) with a typical grain size achieving of $\sim 2 \mu \mathrm{m}$. The grains become essentially equiaxed and typically contained annealing twins (Fig. 1b). Above $200^{\circ} \mathrm{C}$, however, the grain structure was found to change significantly neither in the overburden layer (Figs. 1b-e) nor within the copper lines (Figs. 1f-m). Despite the very fine-grained nature, the microstructure therefore was surprisingly stable; at least, no evidences of the abnormal grain growth were found in the microstructure. It is interesting to note that some grains located in neighboring trenches in the upper part of the lines had nearly the same crystallographic orientation (an example is encircled in Fig. 1i). This effect has already been reported in the literature [8] and may be associated with downward penetration of the growing grains from the overburden layer.

\section{Texture}

Attempting to provide an additional insight into the grain structure, textural data were derived from EBSD data. The figures illustrating the effect of annealing temperature on volume fraction of texture components in the overburden layer, upper and bottom parts of the lines are summarized in Fig. 2.

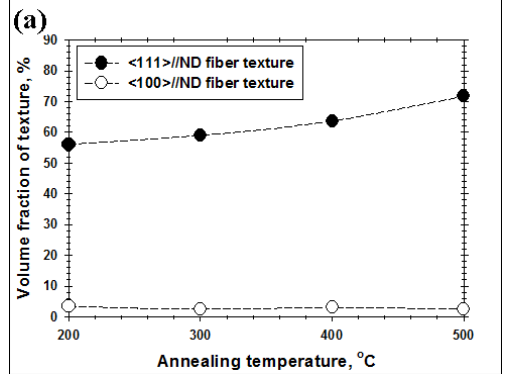

Fig. 2 Effect of annealing temperature on

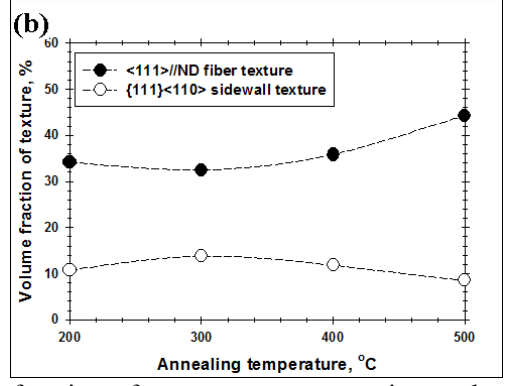

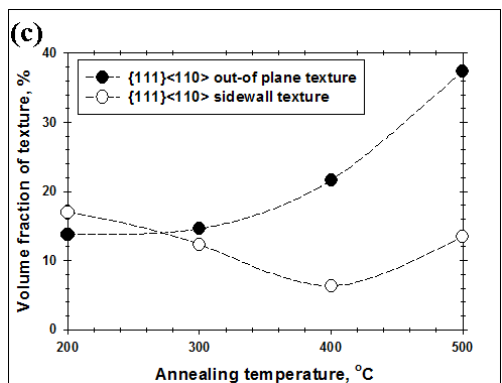

Annealing temperature, ${ }^{\circ} \mathrm{C}$ lines.

In the upper part of the trench as well as in the overburden layer, a notable strengthening of $<111>/ / \mathrm{ND}$ fiber texture is evident with annealing temperature (Figs. 2a and b). This observation may be interpreted in the terms of downward penetration of the growing grains from the overburden into the upper part of the line. On the other hand, a significant increase of the $\{111\}<110>$ out-of plane texture with temperature is evident in the bottom part of the line (Fig. 2c). This may indicate the upward growth of the bottom grains. 
However, though the textural measurements revealed some evidences of the vertical grain growth in the lines, this effect seems to be not very large (Fig. 1).

\section{Discussion}

Grain growth is commonly believed to be driven by a minimization of energy stored in grain boundaries and thus is directly related with grain-boundary area in a material. In this study, the grain boundary area in the overburden layer as well as within the lines was estimated and found to be approximately equal to $\sim 15-20 \mu \mathrm{m} / \mu^{2}$ in all cases (Fig. 3). Considering a principal difference in grain size in these regions $(\sim 2 \mu \mathrm{m}$ in the overburden and $\sim 0.2 \mu \mathrm{m}$ within the line, Fig. 1), this result seems to be very surprising.

This effect is thought to be associated with the specific bamboo-type morphology of the grains within the line. In this case, the longitudinal (i.e. long) boundaries of the grains are phase boundaries (between the copper and tantalum diffusion barrier) whereas transversal (i.e. short) boundaries are grain boundaries (between copper grains). In other words, the microstructure within the lines is characterized by the dominance of phase boundaries whereas the surface area of the grain boundaries is relatively small. As a result, $0.2-\mu \mathrm{m}$ grains within the lines have approximately the same grainboundary area (i.e. grain boundary energy) as $2-\mu \mathrm{m}$ grains in the overburden layer (Fig. 3). This simple idea enables to explain the relatively low thermal stability of the overburden layer as compared to that of the material within the lines. It seems that the grain growth initiates in the overburden layer simply due to larger grain boundary area (and thus higher grain-boundary energy) in this region.

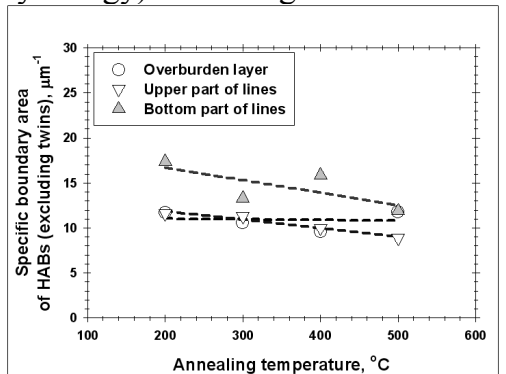

Fig. 3 Effect of annealing temperature on specific grain-boundary area of HABs without $\Sigma 3$ twin boundaries in different parts of lines.

The observed stabilization of the grain structure at nearly the same level of grain-boundary area enables to suggest that the stabilization effect is related with pinning effect of the second phase particles. The secondary particles are thought to be entrapped by the material during electro-deposition process. If the distribution of the secondary particles in the asdeposited material is the same within the overburden and the lines, the grain growth in both cases should be stagnated after achieving of the same grain-boundary area.

\section{Conclusions}

In this work, a structural response of nano-scale damascene copper lines to annealing was studied. To this end the damascene copper was annealed in a practical range of temperatures of $200-500^{\circ} \mathrm{C}$ and microstructural and textural changes were tracked in the overburden layer as well as in the upper and bottom parts of the lines. The main conclusions from this work are as follows:
Experimental observations showed that the microstructure of the overburden layer significantly coarsens during annealing at $200^{\circ} \mathrm{C}$. However, a heat treatment at higher temperatures did not led to substantial changes in grain structure either in the overburden layer or within the line. The textural observations revealed some evidences of the downward penetration of the growing grains from the overburden layer as well as upward growth of the bottom grains. In both cases, however, the grain growth effect was relatively small.

It was deduced that the relatively high thermal stability of the material within the lines may be attributed to the relatively low grain-boundary area in this region. Due to specific bamboo-morphology as well as geometric restrictions of the lines the microstructure here is characterized by the dominance of phase boundaries (between the copper and tantalum diffusion barrier) but not grain boundaries.

The grain structure within the lines as well in the overburden layer was found to stabilize after achieving of nearly the same grain boundary area. This observation was interpreted as indirect evidence that the grain growth in damascene copper lines is controlled by the pinning effect of second-phase particles which presumably were entrapped during the electro-deposition process.

\section{References}

[1] W. Steinhogl, G. Schindler, G. Steinlesberger, M. Traving, M. Engelhardt, "Comprehensive study of the resistivity of copper wires with lateral dimensions of $100 \mathrm{~nm}$ and smaller”, J. Appl. Phys., 97, №2 023706, 2005.

[2] V. Carreau, S. Maîtrejean, M. Verdier, Y. Bréchet, A. Roule, A. Toffoli, V. Delaye, G. Passemard, "Evolution of $\mathrm{Cu}$ microstructure and resistivity during thermal treatment of damascene line: influence of line width and temperature”, Microelectron. Eng., 84, №11, 2007, pp. 27232728.

[3] P.R. Besser, E. Zschech, W. Blum, D. Winter, R. Ortega, S. Rose, M. Herrick, M. Gall, S. Thrasher, M. Tiner, B. Baker, G. Braeckelmann, L. Zhao, C. Simpson, C. Capasso, H. Kawasaki, and E. Weitzman, "Microstructural Characterization of Inlaid Copper Interconnect Lines", J. Electron. Mater., 30, 2001, pp. 320-330.

[4] W. Wu, D. Ernur, S.H. Brongersma, M. Van Hove, K. Maex, "Grain growth in copper interconnect lines”, Microelectron. Eng., 76, 2004, pp. 190-194.

[5] W. Zhang, S.H. Brongersma, N. Heylen, G. Beyer, W. Vandervorst, and K. Maexa, "Geometry Effect on Impurity Incorporation and Grain Growth in Narrow Copper Lines", J. Electrochem. Soc., 152, 2005, pp. C832-C837.

[6] S.H. Brongersma, E. Kerr, I. Vervoort, A. Saerens, K. Maex, "Grain growth, stress, and impurities in electroplated copper”, J. Mater. Res., 17, 2002, pp. 582-589.

[7] H.-J. Lee, D.I. Kim, J.H. Ahn, D.N. Lee, "Electron backscattered diffraction analysis of copper damascene interconnect for ultralargescale integration", Thin Solid Films, 474, 2005, pp. 250-254.

[8] K. Vanstreelsa, S.H. Brongersma, Z. Tokei, L. Carbonell, "Increasing the mean grain size in copper films and features”, J. Mater. Res., 23, №3, 2008, pp. 642-662.

[9] N.-J. Park, D.P. Field, "Predicting thickness dependent twin boundary formation in sputtered $\mathrm{Cu}$ films", Scripta Mater., 54, 2006, pp. 9991003.

[10] Kabirkumar J. MIirpur, and Jerzy A. Szpunar, "Influence of substrate on high-temperature behavior of copper film studied in situ by electron backscatter diffraction", J. Electron. Mater., 34, 2005, pp. 1509-1520.

[11] H. Park, S.-J. Hwang, Y.-C. Joo, "Stress-induced surface damage and grain boundary characteristics of sputtered and electroplated copper thin films", Acta Mater., 52, 2004, pp. 2435-2440.

[12] D.G. Brandon, "The structure of high-angle boundaries", Acta Metall., 14, 1966, pp. 1479-1484.

[13] F.J. Humphreys, "Quantitative metallography by electron backscatter diffraction”, J. Microscopy, 195, 1999, pp. 170-185. 